\title{
ESTUDO ELETRÔNICO DA REATIVIDADE DA 8-HIDROXIQUINOLINA SUBSTITUIIDA FRENTE AO SÍTIO DE LIGAÇÃO DO PEPTÍDEO $\beta$-AMILOIDE AO ÍON ZINCO
}

Talis U. Silva ${ }^{a}$ e Sérgio de Paula Machadoa,*,(D)

a'Departamento de Química Inorgânica, Instituto de Química, Universidade Federal do Rio de Janeiro, 21945-970 Rio de Janeiro - RJ, Brasil

Recebido em 27/06/2019; aceito em 07/08/2019; publicado na web em 11/09/2019

\begin{abstract}
ELECTRONIC STUDY OF REACTIVITY OF THE SUBSTITUTED 8-HYDROXYQUINOLINE TO THE BIND SITE OF THE $\beta$-AMYLOID PEPTIDE TO ZINC ION. In this work, density functional theory (DFT) was used to study the caused effect on reactivity of 8-hydroxyquinoline (8-HQ) by substituent groups (-H, $-\mathrm{CH}_{3},-\mathrm{OCH}_{3},-\mathrm{Cl}$ and $-\mathrm{NO}_{2}$ ) on different positions of the 8-HQ structure. DFT was also used to obtain the electronic profile of $\beta$-amyloid peptide $(\mathrm{A} \beta$ ) bind site to Zinc. The energies and absolute hardness $(\eta)$ values, besides participation percentages values and graphical representation of highest occupied molecular orbital (HOMO) of 8-HQ derivatives and lowest unoccupied molecular orbital (LUMO) of A $\beta$ bind site to Zinc (ZnA $\beta$ ) were analysed. The calculations indicated that the hardest ligands with each substituent formed the most stable complexes with Zinc, and the electronic nature of substituent points to a preferential position on 8-HQ structure. The LUMO profile of ZnA $\beta$ showed that the ligands dislocate one of histidine residues for interacting with Zinc.
\end{abstract}

Keywords: Alzheimer's disease; $\beta$-amyloid; 8-hydroxyquinoline derivatives; complexes of zinc; DFT.

\section{INTRODUÇÃO}

A doença de Alzheimer (DA) é uma doença que causa graves danos neuronais e compromete principalmente a região do hipocampo do cérebro, responsável pelas funções cognitiva e de memória, levando o doente à morte, acometendo, especialmente, a idosos. ${ }^{1} \mathrm{~A}$ DA é uma doença multialvo, na qual diversos fatores parecem contribuir para o seu desenvolvimento, como a diminuição dos níveis do fator de crescimento dos axônios TGF- $\beta 1$ ou da irisina, que é responsável por proteger as sinapses..$^{2,3}$ Entretanto, a principal causa conhecida da doença é oriunda da hipótese da cascata amiloide, em que o acúmulo de $\mathrm{A} \beta$ no cérebro do doente leva à formação das placas senis encontradas na autópsia da vítima e a uma série de outros eventos danosos, como a formação de emaranhados de proteína Tau e estresse oxidativo. ${ }^{1}$

$\mathrm{O}$ A $\beta$ possui em sua estrutura diversos átomos com pares de elétrons livres e, como em sistemas bioinorgânicos, acaba agindo como uma base de Lewis e se coordenando à alguns íons metálicos que naturalmente são encontrados no cérebro, como $\mathrm{Fe}^{3+}, \mathrm{Cu}^{2+} \mathrm{e}^{2+}$, seguindo a tendência de íons metálicos de interagirem com moléculas biológicas. ${ }^{1,4} \mathrm{Os}$ íons $\mathrm{Fe}^{3+}$ e $\mathrm{Cu}^{2+}$ são redoxativos e suas reações com $\mathrm{A} \beta$ acabam por gerar algumas espécies reativas de oxigênio (EROs), como a $\mathrm{H}_{2} \mathrm{O}_{2}$ e o ânion radical superóxido. ${ }^{5} \mathrm{O} \mathrm{Zn}^{2+}$, por outro lado, nao é redox ativo (devido a sua configuração eletrônica $\mathrm{d}^{10}$ ), mas tem papel importante no processo de formação da memória e da função cognitiva, no qual o íon se liga a proteínas específicas nas vesículas sinápticas, como o transportador ZnT3 (que tem sua concentração diminuída em cérebros com DA), originando os impulsos nervosos necessários para a geração da memória. ${ }^{6-8}$

A coordenação de $\mathrm{A} \beta$ ao $\mathrm{Zn}^{2+}$ resulta em um complexo no qual o $\mathrm{Zn}^{2+}$ acaba sendo coordenado pelos resíduos de outra cadeia do peptídeo, em uma reação em cadeia que gera a forma oligomérica nociva do mesmo. ${ }^{9}$ Os resíduos de $\mathrm{A} \beta$ responsáveis por coordenar o $\mathrm{Zn}^{2+}$ em um ambiente tetraédrico estão compreendidos na região 1-16 do peptídeo: Asp1, Glu3, His6, Asp7, Glu11, His13 e His14,

*e-mail: sergiopm@iq.ufrj.br onde, em pH 6,5, o metal é coordenado pelas três histidinas e por Glu11, e em pH 7,4 por His6 e outra histidina, além de Glu11 e um dos aspartatos ou Glu3. ${ }^{10,11}$

Uma abordagem terapêutica contra a DA que envolve os íons metálicos presentes no cérebro é o uso de ligantes capazes de se complexar a esses metais, removendo-os do peptídeo, e derivados da 8-HQ vem sendo propostos como potenciais agentes complexantes dos metais envolvidos na doença, competindo com o peptídeo por, principalmente, $\mathrm{Cu}^{2+}$ e $\mathrm{Zn}^{2+}$. ${ }^{12-14}$

A 8-HQ e seus derivados são uma subclasse das quinolinas que atuam como ligantes bidentados com boa afinidade para íons divalentes de metais de transição, o que justifica seu uso como base

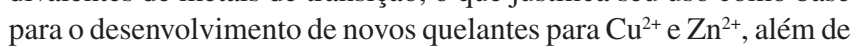
possuírem diversas funções biológicas conhecidas, como fungicida, bactericida e antibacteriana. ${ }^{15}$

O clioquinol (CQ), um derivado da 8-HQ contendo átomos de $\mathrm{Cl}$ e I nas posições 5 e 7, respectivamente, foi um composto pioneiro no uso de quelantes como tratamento para a DA. ${ }^{16}$ Dentro da classe das HQs, compostos do tipo 2-HQ, 4-HQ e 6-HQ não apresentaram desempenho satisfatório em inibir a formação de $\mathrm{A} \beta$ oligoméricos (forma mais tóxica do peptídeo), quando comparado com a 8-HQ e seus derivados, principalmente derivados halogenados, como o CQ. ${ }^{17}$

Uma vez que a 8-HQ é uma importante base para síntese de potencias quelantes para o tratamento da DA, torna-se fundamental estudar sua reatividade e a de seus derivados frente ao peptídeo complexado à íons relevantes na $\mathrm{DA}$, como o $\mathrm{Zn}^{2+}$. Assim, neste trabalho, cálculos de DFT foram empregados para verificar como grupos de diferentes naturezas eletrônicas influem na reatividade da 8-HQ frente ao sítio $\mathrm{ZnA} \beta$ e como a posição do substituinte influencia na estabilidade dos complexos formados.

\section{METODOLOGIA}

As estruturas otimizadas foram obtidas em fase gasosa por meio de cálculos de DFT, utilizando o funcional B3LYP. ${ }^{18,19}$ O conjunto de bases LanL2DZ foi empregado, ${ }^{20} \mathrm{o}$ qual aplica um pseudopotencial no átomo de zinco e utiliza a base D95V para os demais átomos. O nível 
B3LYP/LanL2DZ foi empregado em um trabalho anterior envolvendo complexos de $\mathrm{Zn}^{2+}$ com derivados da 8-HQ, apresentando excelentes resultados. ${ }^{21}$ Todos os cálculos foram realizados no programa Gaussian 09.22 Foram realizados cálculos de frequência nas estruturas otimizadas obtidas, e a ausência de frequências imaginárias nessas estruturas confirmou que essas representaram o mínimo de energia. Os valores de energia obtidos representam a energia eletrônica com a correção de ponto de zero. Os valores de dureza $(\eta)$ para as estruturas foram obtidos por meio da Eq. 1, conforme proposto por Parr e Pearson, ${ }^{23}$ em que: $\mathrm{E}_{\mathrm{Lumo}}$ e $\mathrm{E}_{\mathrm{Homo}}$ são as energias dos orbitais de fronteira $L U M O$ e $H O M O$, respectivamente.

$$
\eta=\frac{E_{L U M O}-E_{H O M O}}{2}
$$

Também foram obtidos os coeficientes de participação de cada átomo dos ligantes no orbital HOMO e de cada átomo dos complexos $\mathrm{ZnA} \beta$ no orbital LUMO por meio da palavra-chave pop=full, para posterior cálculo dos percentuais de participação de átomos selecionados nesses orbitais.

\section{RESULTADOS E DISCUSSÃO}

\section{Estudo dos sítios ZnA $\beta$}

$\mathrm{Na}$ estrutura experimental obtida por Zirah e colaboradores, em $\mathrm{pH} 6,5,{ }^{11} \mathrm{o} \mathrm{Zn}{ }^{2+}$ é coordenado por três resíduos de histidina e um glutamato, enquanto, no trabalho de Alies e colaboradores, em $\mathrm{pH} 7,4,{ }^{10}$ a coordenação se dá por dois resíduos de histidina, um glutamato e um outro resíduo com terminação carboxilada (Asp1, Glu3 ou Asp7). Em trabalhos teóricos, também são feitas propostas para o sítio ZnA $\beta$, como no trabalho de Marino e colaboradores, ${ }^{24}$ em que a estrutura mais estável obtida é similar ao sítio ZnA $\beta$ de Zirah e colaboradores, com $\mathrm{Zn}^{2+}$ coordenado tetraedricamente por três átomos de nitrogênio (N) de anéis de 4-metil-imidazol e um oxigênio $(\mathrm{O})$ de um grupo

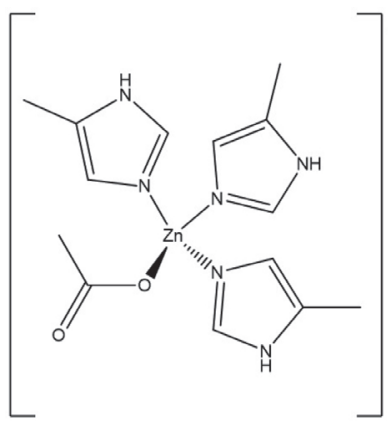

$\operatorname{ZnA} \beta(a)$<smiles></smiles>

$\operatorname{ZnA\beta (b)}$
Figura 1. Estruturas propostas neste estudo para o sítio ZnA $\beta$

acetato. Assim, dois modelos foram utilizados no presente estudo para mimetizar o sítio $\mathrm{ZnA} \beta$, um simulando o sítio proposto por Zirah e colaboradores e o outro simulando o sítio proposto por Alies e colaboradores, $\mathrm{ZnA} \beta$ (a) e $\mathrm{ZnA} \beta$ (b), respectivamente (Figura 1).

Foram obtidas as estruturas otimizadas de cada complexo, bem como o diagrama de contorno de seus orbitais LUMO (Figura 2), que será o orbital a receber densidade eletrônica, por meio dos orbitais HOMO dos ligantes derivados da 8-HQ.

Como se pode observar na Figura 2, o orbital LUMO, em ambos os modelos, possui contribuição majoritária de um dos ligantes, um dos grupos 4-metil-imidazol. Isso indica que, no sítio de coordenação do complexo natural de $\mathrm{A} \beta$ com $\mathrm{Zn}^{2+}$, um dos resíduos de histidina possui contribuição majoritária no LUMO. Isso pode ser visualizado de maneira quantitativa quando se observa os valores de percentuais de participação nesse orbital para ambos os modelos (Tabela 1).

Como se confirma na Tabela 1, o grupo 4-metil-imidazol com contribuição majoritária no $L U M O$ possui contribuição superior a $65 \%$ em ambos os complexos, em que a contribuição em $\mathrm{ZnA} \beta(\mathrm{b})$ é cerca de $10 \%$ superior do que a em $\mathrm{ZnA} \beta$ (a). Em contrapartida, a contribuição de $\mathrm{Zn}^{2+}$ nesse orbital é inferior a 3\%, ou seja, a interação do HOMO dos ligantes não se dará com o íon metálico e sim com

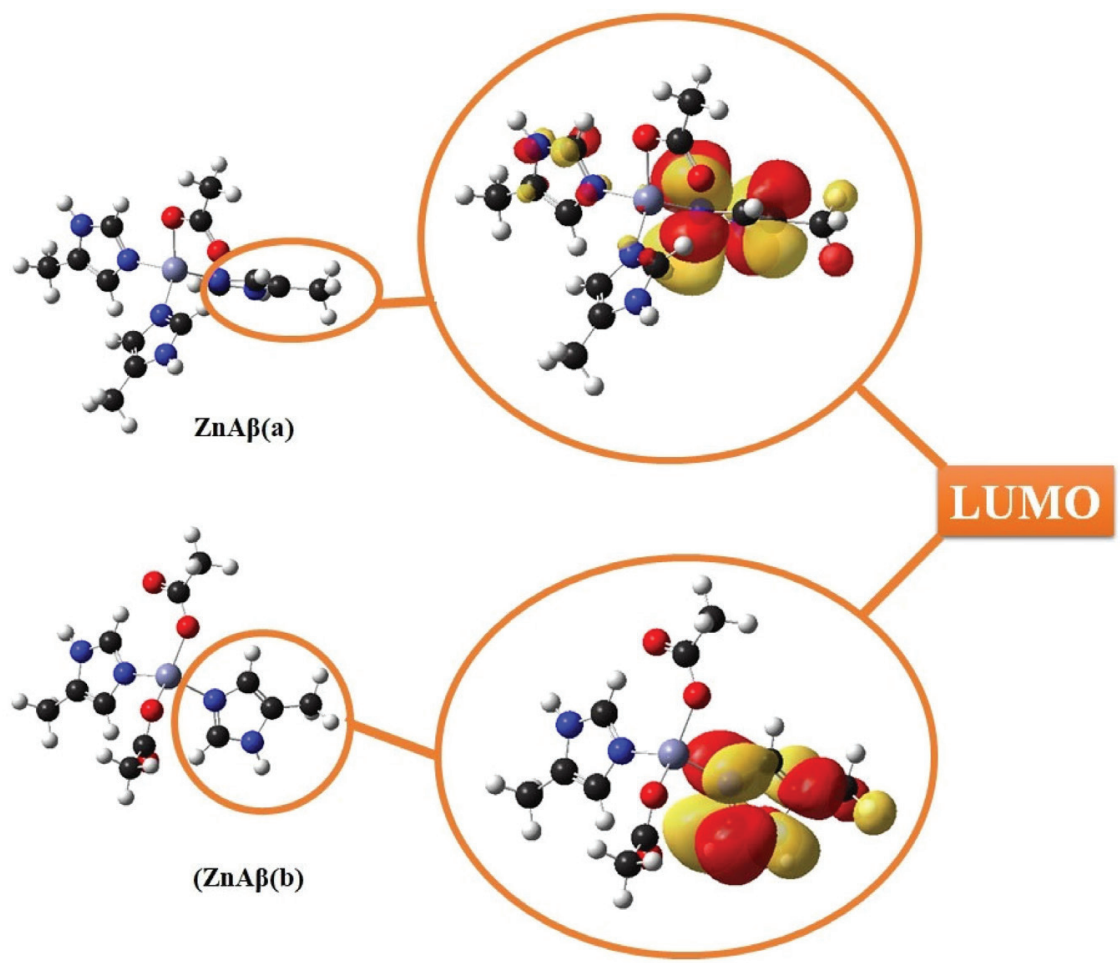

Figura 2. Estruturas otimizadas obtidas para os modelos do sítio ZnA e diagramas de contorno de seus orbitais LUMO. Em destaque, os grupos 4-metil-imidazol de cada sítio que representam o LUMO de cada sítio 
Tabela 1. Valores de $\eta$ para cada modelo e percentuais de participação no orbital LUMO (\% LUMO) de $\mathrm{Zn}^{2+}$ e do grupo 4-metil-imidazol com maior contribuição do $L U M O$

\begin{tabular}{lcc}
\hline Modelo & $\mathrm{ZnA} \beta(\mathrm{a})$ & $\mathrm{ZnA} \beta(\mathrm{b})$ \\
\hline$\eta(\mathrm{eV})$ & 3,11 & 2,91 \\
$\%$ LUMO do 4-metil-imidazol & 69,9 & 79,4 \\
$\%$ LUMO do $\mathrm{Zn}^{2+}$ & 2,84 & 2,65 \\
\hline
\end{tabular}

o grupo 4-metil-imidazol, indicando que o provável mecanismo de ação de um ligante para remover o íon do sítio do peptídeo envolve o deslocamento de um dos resíduos de histidina da esfera de coordenação e não um ataque direto ao centro metálico. É possível traçar um paralelo entre esses resultados e os resultados obtidos por Tsvetkov e colaboradores ${ }^{25} \mathrm{em}$ que os autores identificaram, por meio de cálculos de dinâmica molecular, os resíduos Glu11, His13 e His14 como responsáveis pelo reconhecimento do $\mathrm{Zn}^{2+}$ no meio extracelular e por coordená-lo. A esfera de coordenação tetraédrica seria completa, em um primeiro momento, por uma molécula de água, e, logo em seguida, ela seria substituída por His6, mostrando que His6 não faz parte do sítio mínimo de ligação ao $\mathrm{Zn}^{2+}$. Como a região dos modelos de $\mathrm{ZnA} \beta$ que deve interagir com a 8-HQ e seus derivados se mostrou ser um resíduo de histidina (representado pelo grupo 4-metil-imidazol de maior contribuição no $L U M O$ ), é possível que esse resíduo seja justamente His6, que será removido primeiramente da esfera de coordenação por influência do ligante por não fazer parte do sítio mínimo de ligação do $\mathrm{Zn}^{2+}$.

A Tabela 1 também apresenta os valores de $\eta$ para os dois modelos. De acordo com o enunciado de Pearson, ${ }^{26}$ quanto mais próxima for a dureza entre duas espécies, melhor se dará a interação entre elas e maior será a estabilidade do produto formado. $\mathrm{O}$ valor de $\eta$ encontrado para os dois complexos foi bem próximo, com um valor médio de 3,01, o que indica que, quanto mais próximo for o valor de $\eta$ encontrado para os ligantes derivados da 8-HQ, maior será a estabilidade do complexo formado desses ligantes com o $\mathrm{Zn}^{2+}$.

\section{Estudo dos ligantes e complexos de derivados da 8-HQ}

Foram propostos seis modelos para quatro ligantes derivados da 8-HQ, totalizando 25 estruturas (incluindo a 8-HQ). A Figura 3 apresenta as estruturas genéricas para todos os ligantes com o substituintes X.

Os principais parâmetros geométricos para os ligantes de modelo f são apresentados na Tabela 1S (Material Suplementar) e são comparados com os valores da estrutura experimental da 8-HQ HXQUIN11 obtida no Cambridge Structural Database (CSD). ${ }^{27}$ Esse modelo foi escolhido para análise por ser o único a apresentar diferença significativa na região de coordenação, com erros relativos máximos de $5 \%$. Para os demais modelos, os erros foram inferiores a 3\%, indicando boa concordância nos resultados.

Foram obtidos os valores de $\eta$ para todos os 25 ligantes deste estudo, que podem ser vistos na Tabela $2 \mathrm{~S}$, no Material Suplementar. Uma vez que os valores obtidos foram relativamente próximos para as estruturas com o mesmo substituinte, foram tomados então os ligantes mais duros para cada substituinte para avaliação e comparação com a 8-HQ. A Tabela 2 apresenta os valores de $\eta$ para os ligantes de maior $\eta$ para cada substituinte, enquanto suas estruturas otimizadas obtidas são apresentadas na Figura 4.

Como se pode observar pelos dados da Tabela 2 e estruturas da Figura 4, o mesmo modelo não apresentou o maior valor de $\eta$ para todos os substituintes. Ao invés disso, para cada substituinte houve uma posição específica onde se obteve o ligante mais duro,<smiles>[X]c1cnc2c(O)cccc2c1</smiles>

a

b

c<smiles>[X]c1ccc(O)c2ncccc12</smiles>

d<smiles>[X]c1cc(O)c2ncccc2c1</smiles>

e<smiles>[X]c1ccc2cccnc2c1O</smiles>

f

$$
\left.\left.\mathrm{X}=\mathrm{H}_{\mathrm{H}}\right\}_{\mathrm{CH}_{3}}\right\}_{\mathrm{Cl}}
$$

Figura 3. Estruturas genéricas para os ligantes derivados da 8-HQ com substituinte $-\mathrm{X}, \mathrm{com} \mathrm{X}=-\mathrm{H},-\mathrm{OCH}_{3},-\mathrm{CH}_{3},-\mathrm{Cl} e-\mathrm{NO}_{2}$

Tabela 2. Valores de $\eta(\mathrm{eV})$ para os ligantes mais duros com cada substituinte

\begin{tabular}{lcc}
\hline Ligante & Modelo & $\eta(\mathrm{eV})$ \\
\hline $8-\mathrm{HQ}$ & - & 2,23 \\
$8-\mathrm{HQ}_{-}-\mathrm{OCH}_{3}$ & c & 2,27 \\
$8-\mathrm{HQ}-\mathrm{Cl}$ & e & 2,25 \\
$8-\mathrm{HQ}-\mathrm{CH}_{3}$ & a & 2,26 \\
$8-\mathrm{HQ}_{-} \mathrm{NO}_{2}$ & f & 1,92 \\
\hline
\end{tabular}

indicando que a natureza eletrônica doadora/aceptora do substituinte indicará sua posição na estrutura da 8-HQ para obtenção do ligante mais duro: doadores $\sigma\left(-\mathrm{CH}_{3}\right)$ na posição orto ao $\mathrm{N}$; doadores $\pi$ e retiradores $\sigma\left(-\mathrm{OCH}_{3}\right)$ na posição para ao $\mathrm{N}$; doadores $\pi$ e fortes retiradores $\sigma-(-\mathrm{Cl})$ na posição meta ao $\mathrm{O}$; e fortes retiradores $\pi$ e $\sigma\left(-\mathrm{NO}_{2}\right)$ na posição orto ao O. Entre os ligantes da Figura 3, o que se apresentou como mais duro foi o $8-\mathrm{HQ}-\mathrm{OCH}_{3}(\mathrm{c})$ e o mais macio foi o 8-HQ- $\mathrm{NO}_{2}$ (f). De acordo com os dados obtidos, os valores de $\eta$ encontrados para cada substituinte ficaram nas seguintes faixas: 2,27-2,07 eV para 8-HQ- $\mathrm{OCH}_{3} ; 2,25-2,09 \mathrm{eV}$ para 8-HQ-Cl; 2,26-2,06 eV para 8-HQ- $\mathrm{CH}_{3}$; e 1,92-1,68 eV para 8-HQ-NO

Para cada ligante da Figura 4, foi obtido o diagrama de contorno do orbital HOMO (Figura 5) e também os percentuais de participação nesse orbital dos átomos coordenantes de cada ligante, que podem ser vistos no Material Suplementar (Tabelas 3S, 4S, 5S, 6S e 7S).

Os orbitais HOMO, para cada ligante, apresentaram caráter predominantemente ligante e perfis bastante semelhantes. Nos átomos de $\mathrm{O}$ e N, esse orbital se apresenta como o par de elétrons não ligante desses átomos, que serão responsáveis por coordenar o íon $\mathrm{Zn}^{2+}$. Os percentuais de participação nesse orbital do $\mathrm{N} \mathrm{e} \mathrm{O}$ da 8-HQ foram 8,25 e 15,3, respectivamente, e valores próximos a esses foram obtidos para os demais ligantes. Os valores se encontraram dentro das seguintes faixas: 6,81-8,37\% (N) e 14,4-15,2\% (O) para 8-HQ- $\mathrm{CH}_{3}$; $5,83-9,50 \%(\mathrm{~N})$ e $10,3-13,3 \%(\mathrm{O})$ para $8-\mathrm{HQ}-\mathrm{OCH}_{3} ; 7,02-8,16 \%(\mathrm{~N})$ e 13,6-14,9 (O) para 8-HQ-Cl; e 6,66-8,61\% (N) e 14,7-16,6\% (O) para 8-HQ- $\mathrm{NO}_{2}$. No geral, os ligantes que apresentaram maior contribuição no $H O M O$ na região de coordenação foram os de modelo f.

Foram obtidas as estruturas otimizadas dos complexos de $\mathrm{Zn}^{2+}$ com cada um dos ligantes estudados, e todos apresentaram geometria 

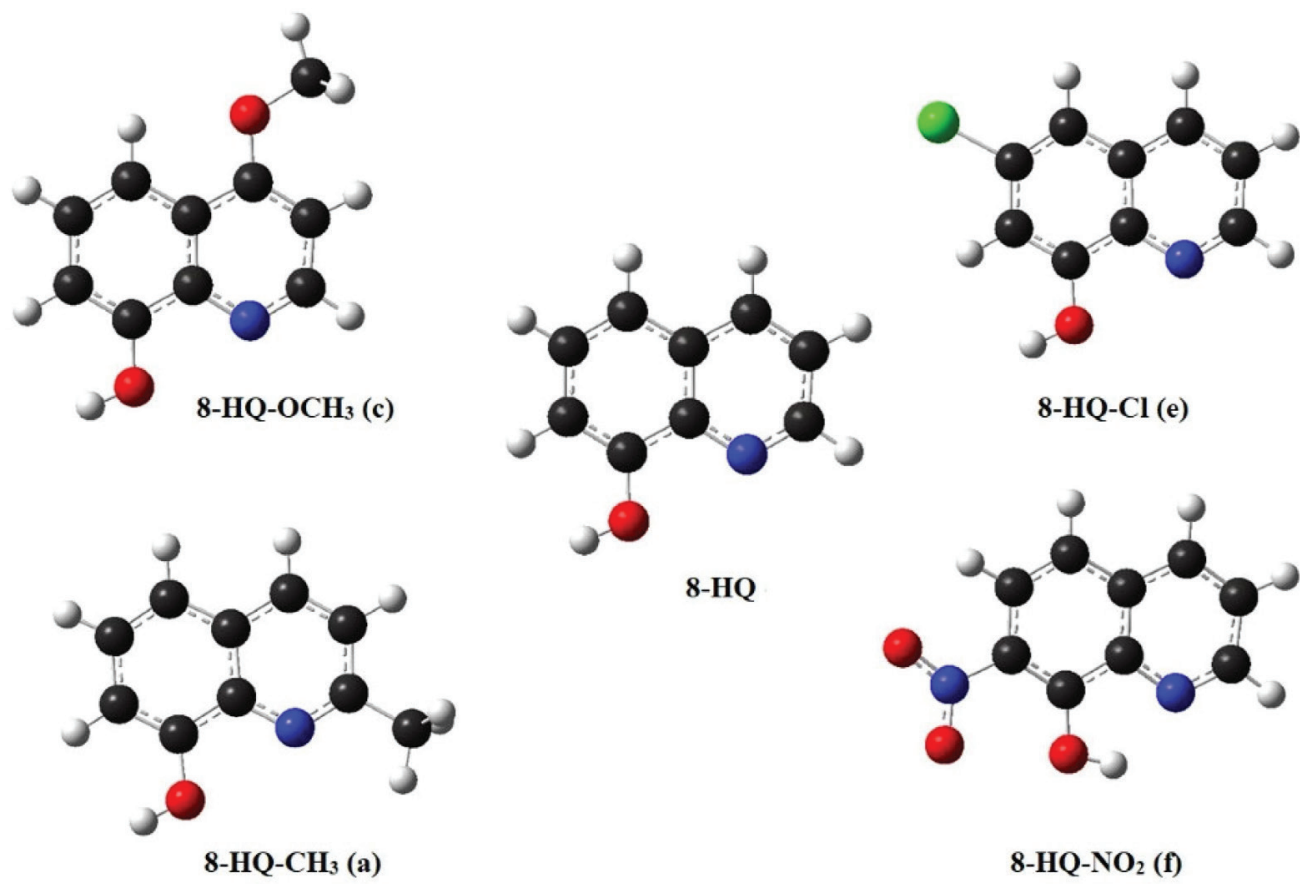

8-HQ-NO 2 (f)

Figura 4. Estrutura otimizadas dos ligantes de maior $\eta$ com cada substituinte

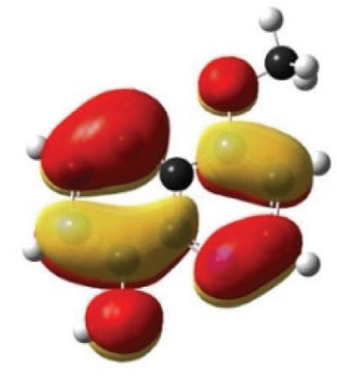

8-HQ- $\mathrm{OCH}_{3}$ (c)

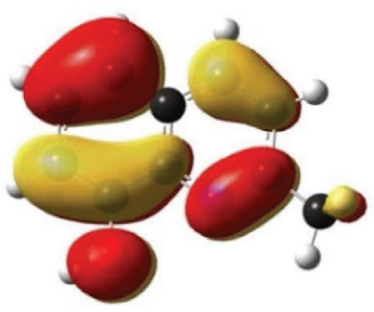

8-HQ- $\mathrm{CH}_{3}$ (a)

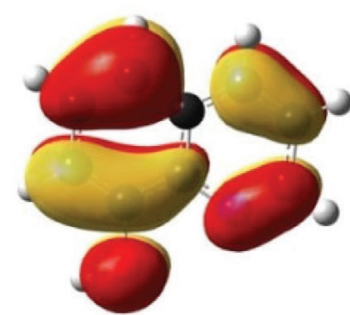

8-HQ

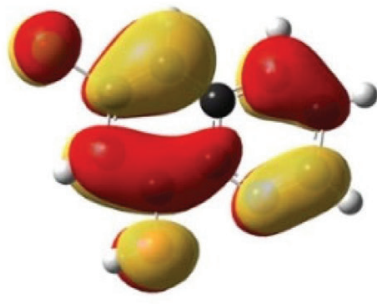

8-HQ-Cl (e)

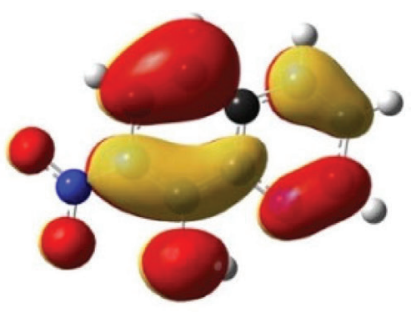

8-HQ-NO 2 (f)

Figura 5. Diagramas de contorno do orbital HOMO para cada ligante da Figura 3

tetrédrica em torno do centro de coordenação. Suas energias relativas são apresentadas na Tabela 3. Já a Figura 6, apresenta os estruturas otimizadas para os complexos mais estáveis obtidos com cada substituinte.

Os valores de energia encontrados mostram que os complexos mais estáveis com cada substituinte foram obtidos a partir dos ligantes de maior $\eta$, com exceção dos ligantes do tipo 8-HQ- $\mathrm{NO}_{2}$, cujo complexo mais estável foi o de modelo d e o ligante de maior $\eta$ foi o de modelo $\mathrm{f}$. Entretanto, no ligante de modelo f, como se observa na Figura 1S (Material Suplementar), um dos átomos de O do substituinte pode se coordenar ao $\mathrm{Zn}^{2+}$ juntamente com o $\mathrm{O}$ fenólico, formando um possível anel de seis membros com o metal, enquanto, para os demais ligantes, só é possível a formação de um anel de cinco membros, menos estável. As distâncias do $\mathrm{O}$ quinolínico ao $\mathrm{O}$ do substituinte (2,69 ̊) e ao N quinolínico (2,63 Å) são bem próximas, o que também indica que a coordenação por ambos os modos é possível. Essa nova estrutura (modelo f.1, Figura 6) foi também obtida pelos cálculos e comparada em energia aos modelos $\mathrm{f}$ (com coordenação via $\mathrm{N}$ e $\mathrm{O}$ quinolínicos) e com o modelo d (Tabela 3).

Como se pode observar nas Tabelas 3 e 4, os ligantes de maior dureza com cada substituinte foram aqueles que geraram os complexos mais estáveis, mesmo para o substituinte $-\mathrm{NO}_{2}$, no qual houve a mudança do sítio e da coordenação. Isso está de acordo com o enunciado de Pearson, ${ }^{26}$ em que quanto mais próxima foi a dureza 
Tabela 3. Energias relativas $\left(\mathrm{kcal} \mathrm{mol}^{-1}\right)$ para os isômeros de cada complexo

\begin{tabular}{ccccc}
\hline Modelo & {$\left[\mathrm{Zn}\left(8-\mathrm{HQ}-\mathrm{OCH}_{3}\right)_{2}\right]$} & {$\left[\mathrm{Zn}(8-\mathrm{HQ}-\mathrm{Cl})_{2}\right]$} & {$\left[\mathrm{Zn}\left(8-\mathrm{HQ}-\mathrm{CH}_{3}\right)_{2}\right]$} & {$\left[\mathrm{Zn}\left(8-\mathrm{HQ}-\mathrm{NO}_{2}\right)_{2}\right]$} \\
\hline a & 0,325 & 3,41 & 0,0 & 3,92 \\
b & 6,55 & 5,54 & 4,76 & 15,6 \\
c & 0,0 & 3,42 & 3,69 & 18,8 \\
d & 8,33 & 2,28 & 8,23 & 0,0 \\
e & 2,66 & 0,0 & 4,55 & 4,48 \\
$\mathrm{f}$ & 15,34 & 3,95 & 4,28 & 9,41 \\
\hline
\end{tabular}

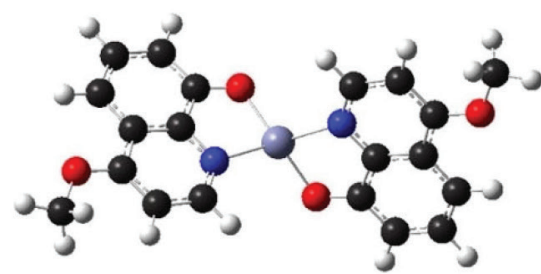

$\left[\mathrm{Zn}\left(8-\mathrm{HQ}_{\mathbf{Q}}-\mathrm{OCH}_{3}\right)_{2}\right]$ (c)

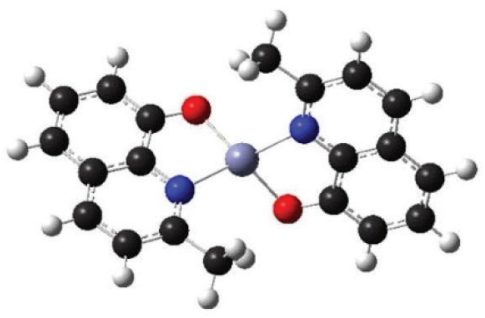

$\left[\mathrm{Zn}\left(\mathbf{8}-\mathrm{HQ}-\mathrm{CH}_{3}\right)_{2}\right]($ a)
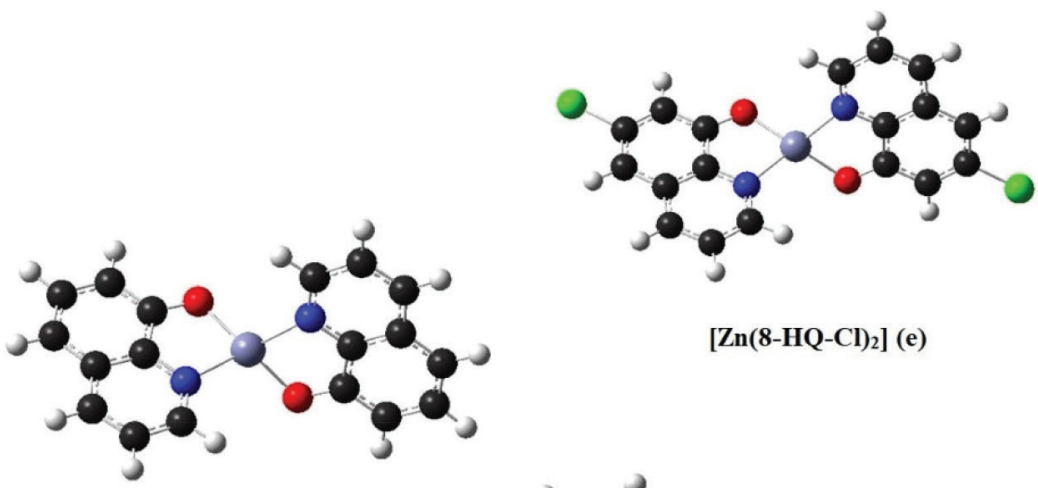

[Zn(8-HQ-Cl) $)_{2}(\mathrm{e})$

$\left[\mathrm{Zn}(\mathbf{8}-\mathrm{HQ})_{2}\right]$

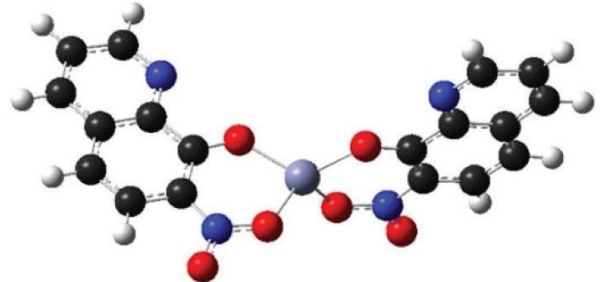

$\left[\mathrm{Zn}\left(\mathbf{8}-\mathrm{HQ}-\mathrm{NO}_{2}\right)_{2}\right](\mathbf{f . 1})$

Figura 6. Estruturas otimizadas dos complexos com os ligantes de maior $\eta$ com cada substituinte

Tabela 4. Energias relativas $\left(\mathrm{kcal} \mathrm{mol}^{-1}\right)$ para os isômeros do complexo com substituinte $-\mathrm{NO}_{2}$

\begin{tabular}{cc}
\hline Modelo & Energia relativa \\
\hline $\mathrm{d}$ & 8,31 \\
$\mathrm{f}$ & 17,7 \\
$\mathrm{f} .1$ & 0,0 \\
\hline
\end{tabular}

dos ligantes de cada classe de substituintes da dureza do sítio $\mathrm{ZnA} \beta$, maior foi a estabilidade do produto formado.

Assim como feito para o ligante 8-HQ- $\mathrm{NO}_{2}$ de modelo f, para os outros dois ligantes com pares de elétrons livres na posição orto ao $\mathrm{O}$ (modelo $\mathrm{f}$ ), ou seja, 8-HQ- $\mathrm{OCH}_{3}, 8-\mathrm{HQ}-\mathrm{Cl}$, foram obtidas as estruturas otimizadas para complexos de modelo f.1 com esses dois ligantes (Figura 1S, Material Suplementar), entretanto, as estruturas apresentaram energia mais alta do que seus respectivos isômeros. Para [Zn(8-HQ- $\left.\mathrm{OCH}_{3}\right)_{2}$ ], o modelo f.1 apresentou 20,1 kcal mol ${ }^{-1}$ a mais em energia do que o modelo f e também apresentou geometria quadrática plana em torno do íon metálico, mais instável quando comparada à geometria tetraédrica do modelo f. Já para o complexo [ $\left.\mathrm{Zn}(8-\mathrm{HQ}-\mathrm{Cl})_{2}\right]$, o modelo $\mathrm{f} .1$ apresentou energia superior ao modelo f em $45,8 \mathrm{kcal} \mathrm{mol}^{-1}$, indicando que o átomo de $\mathrm{Cl}$ não se coordena ao íon metálico.

Na Figura 6, em todos os complexos se observou um ambiente tetraédrico em torno do $\mathrm{Zn}^{2+}$, e, com exceção de $-\mathrm{NO}_{2}$, o íon metálico formou um anel de cinco membros com o $\mathrm{O}$ e $\mathrm{N}$ quinolínicos e os átomos de $\mathrm{C}$ diretamente ligados aos átomos quelantes. Já para o $-\mathrm{NO}_{2}$, se observou a formação de um anel de seis membros, mais estável, o que explica o porquê desse modelo ser mais estável do que os modelos de f para o complexo com o 8-HQ- $\mathrm{NO}_{2}$.

\section{CONCLUSÃO}

Os resultados obtidos nesse estudo permitiram demonstrar que a formação de complexos de derivados da 8-HQ com substituintes $-\mathrm{CH}_{3},-\mathrm{OCH}_{3},-\mathrm{Cl}$ e $-\mathrm{NO}_{2}$ em fase gasosa está de acordo com teoria de ácidos e bases duros e macios de Pearson, na qual os ligantes de cada classe de substituintes que apresentaram valor de $\eta$ mais próximo dos valores de $\eta$ encontrados para os modelos do sítio $\mathrm{ZnA} \beta$ formaram os complexos mais estáveis com o íon $\mathrm{Zn}^{2+}$. A natureza doadora/aceptora eletrônica do substituinte indica que há uma posição específica na estrutura da 8-HQ que gera o complexo mais estável com $\mathrm{Zn}^{2+}$. A análise qualitativa e quantitativa da contribuição no orbital $L U M O$ revelou que um dos resíduos de histidina do centro de coordenação possui contribuição majoritária nesse orbital (superior a $65 \%$ ), enquanto $\mathrm{Zn}^{2+}$ possui contribuição significativamente baixa (inferior a 3\%), indicando que um agente quelante que remove o íon metálico do peptídeo não interage diretamente com o $\mathrm{Zn}^{2+}$, e sim com o referido resíduo de histidina, que pode possivelmente ser o resíduo His6, o qual não faz parte do sítio mínimo de ligação desse metal no peptídeo. ${ }^{25}$ Com esses resultados, espera-se auxiliar no desenvolvimento de novos derivados da 8-HQ como potenciais agentes quelantes para o $\mathrm{Zn}^{2+}$ complexado ao $\mathrm{A} \beta$ na terapia da $\mathrm{DA}$. 


\section{MATERIAL SUPLEMENTAR}

Algumas imagens e tabelas dos sistemas utilizados neste trabalho estão disponíveis em http://quimicanova.sbq.org.br, na forma de arquivo PDF, com acesso livre.

\section{AGRADECIMENTOS}

Este estudo foi financiado em parte pela Coordenação de Aperfeiçoamento de Pessoal de Nível Superior (CAPES) (Código Financeiro 001; Bolsas de Doutorado para TU da Silva). Os autores agradecem também à Fundação de Amparo à Pesquisa do Estado do Rio de Janeiro (FAPERJ) (bolsa E-26/010.001003/2016) e ao Conselho Nacional de Desenvolvimento Científico e Tecnológico (CNPq) (outorga 304402/2017).

\section{REFERÊNCIAS}

1. De Falco, A.; Cukierman, D. S.; Hauser-Davis, R. A.; Rey, N. A.; Quim. Nova 2016, 39, 63.

2. Diniz, L. P.; Tortelli, V.; Matias, I. C. P.; Morgado, J.; Araújo, A. P. B.; Melo, H. M.; Silva, G. S. S.; Alves-Leon, S. V.; Marcondes, J.; Ferreira, S. T.; De Felice, F. G.; Gomes, F. C. A.; J. Neurosci. 2017, 1, 1.

3. Lourenço, M. V.; Frozza R. L.; de Freitas, G. B.; Zhang, H.; Kincheski, G. C.; Ribeiro, F. C.; Gonçalves, R. A.; Clarke, J. R.; Beckman, D.; Staniszewski, A.; Berman, H.; Guerra, L. A.; Forny-Germano, L.; Meier, S.; Wilcock, D. M.; de Souza, J. M.; Alves-Leon, S.; Prado, V. F.; Prado, M. A. M.; Abisambra, J. F.; Tovar-Moll, F.; Mattos, P.; Arancio, O.; Ferreira, S. T.; De Felice, F.; Nat. Med. 2019, 25, 165.

4. Benite, A. M. C.; Machado, S. P.; Barreiro, E. J.; Quim. Nova 2007, 30, 2062.

5. Mamelak, M.; Neurobiol. Aging 2007, 28, 1340

6. Colvin, R. A.; Fontaine, C. P.; Laskowski, M.; Thomas, D.; Eur. J. Pharmacol. 2003, 479, 171.

7. Sekler, I.; Sensi, S. L.; Hershfinkel, M.; Silverman, W. F.; Mol. Med. 2007, 13, 337 .

8. Roberts, B. R.; Ryan, T. M.; Bush, A. I.; Masters, C. L.; Duce, J. A.; J. Neurochem. 2012, 120, 149.

9. Istrate, A. N.; Kozin, S. A.; Zhokhov, S. S.; Mantsyzov, A. B.; Kechko, O. I.; Pastore, A.; Makarov, A. A.; Polshakov, V. I.; Sci. Rep. 2016, 6, 21734.

10. Alies, B.; Conte-Daban, A.; Sayen, S.; Collin, F.; Kieffer, I. Guillon, E.; Faller, P. Hureau, C.; Inorg. Chem. 2016, 55, 10499.
11. Zirah, S.; Kozin, S. A.; Mazur, A. K.; Blond, A.; Cheminant, M.; Segalas-Milazzo, L.; Debey, P.; Rebuffat, S.; J. Biol. Chem. 2006, 281, 2151.

12. De Freitas, L. V.; Dos Santos, A. L. S. F.; Da Costa, F. C.; Calixto, J. B.; Miranda, P. V. P.; Silva, T. J. J.; Pereira, E. S.; Rocha, W. R.; De Almeida, W. B.; De Souza, L. A.; Freitas, M. C. R.; J. Mol. Struct. 2018, 1169, 119.

13. Freitas, L. V.; Silva, C. C. P.; Ellena, J.; Costa, L. A. S.; Rey, N. A.; Spectrochim. Acta, Part A 2013, 116, 41.

14. Gomes, L. M.; Vieira, R. P.; Jones, M. R.; Wang, M. C.; Dyrager, C.; Souza-Fagundes, E. M.; Da Silva, J. G.; Storr, T.; Beraldo, H.; J. Inorg. Biochem. 2014, 139, 106.

15. Olivieri, V.; Vecchio, G.; Eur. J. Med. Chem. 2016, 120, 252.

16. Melov, S.; Trends Neurosci. 2002, 25, 121.

17. Levine III, H.; Ding, Q.; Walker, J. A.; Voss, R. S.; Augelli-Szafran, C. E.; Neurosci. Lett. 2009, 465, 99.

18. Becke, A.; J. Chem. Phys. 1993, 98, 5648.

19. Lee, C.; Yang, W.; Parr, R. G.; Phys. Rev. B 1988, 37, 785.

20. Hay, P. J.; Wadt, W. R.; J. Chem. Phys. 1985, 82, 270.

21. Silva, T. U.; Freitas, L. V.; Rey, N. A.; Machado, S. P.; Quim. Nova 2018, 41, 1132.

22. Frisch, M. J.; Trucks, G. W.; Schlegel, H. B.; Scuseria, G. E.; Robb, M. A.; Cheeseman, J. R.; Scalmani, G.; Barone, V.; Petersson, G. A.; Nakatsuji, H.; Li, X.; Caricato, M.; Marenich, A.; Bloino, J.; Janesko, B. G.; Gomperts, R.; Mennucci, B.; Hratchian, H. P.; Ortiz, J. V.; Izmaylov, A. F.; Sonnenberg, J. L.; Williams-Young, D.; Ding, F.; Lipparini, F.; Egidi, F.; Goings, J.; Peng, B.; Petrone, A.; Henderson, T.; Ranasinghe, D.; Zakrzewski, V. G.; Gao, J.; Rega, N.; Zheng, G.; Liang, W.; Hada, M.; Ehara, M.; Toyota, K.; Fukuda, R.; Hasegawa, J.; Ishida, M.; Nakajima, T.; Honda, Y.; Kitao, O.; Nakai, H.; Vreven, T.; Throssell, K.; Montgomery, J. A.; Jr.; Peralta, J. E.; Ogliaro, F.; Bearpark, M.; Heyd, J. J.; Brothers, E.; Kudin, K. N.; Staroverov, V. N.; Keith, T.; Kobayashi, R.; Normand, J.; Raghavachari, K.; Rendell, A.; Burant, J. C.; Iyengar, S. S.; Tomasi, J.; Cossi, M.; Millam, J. M.; Klene, M.; Adamo, C.; Cammi, R.; Ochterski, J. W.; Martin, R. L.; Morokuma, K.; Farkas, O.; Foresman, J. B.; Fox, D. J.; Gaussian 09, Revision-B.01-SMP, Gaussian, Inc., Wallingford CT, 2009

23. Parr, R. G.; Pearson, R. G.; J. Am. Chem. Soc. 1983, 105, 7512.

24. Marino, T.; Russo, N.; Toscano, M.; Pavelka, M.; Interdiscip. Sci.: Comput. Life Sci. 2010, 2, 57.

25. Tsvetkov, P. O.; Kulikova, A. A.; Golovin, A. V.; Tkachev, Y. V.; Archakov, A. I.; Kozin, S. A.; Makarov, A. A.; Biophys. J. 2010, 99, 84.

26. Pearson, R. G.; J. Am. Chem. Soc. 1963, 85, 1963.

27. https://www.ccdc.cam.ac.uk, acessada em agosto 2019. 